\title{
Endoscopic Ultrasound-Guided Fine Needle Biopsy Needles Provide Higher Diagnostic Yield Compared to Endoscopic Ultrasound- Guided Fine Needle Aspiration Needles When Sampling Solid Pancreatic Lesions: A Meta-Analysis
}

\author{
Benjamin D. Renelus ${ }^{1}$, Daniel S. Jamorabo ${ }^{1}$, Iman Boston ${ }^{2}$, William M. Briggs ${ }^{3}$ and John M. Poneros ${ }^{4}$ \\ ${ }^{1}$ Division of Gastroenterology and Hepatobiliary Disease, New York-Presbyterian Brooklyn Methodist Hospital, Brooklyn, NY, \\ ${ }^{2}$ Department of Internal Medicine, Wright State University, Dayton, OH, ${ }^{3}$ Department of Epidemiology and Biostatistics, Weill Cornell \\ Medical College, New York, NY, ${ }^{4}$ Division of Gastroenterology and Hepatology, New York Presbyterian, Columbia University Medical \\ Center, New York, NY, USA
}

Background/Aims: Studies comparing the utility of endoscopic ultrasound-guided fine needle aspiration (EUS-FNA) and endoscopic ultrasound-guided fine needle biopsy (EUS-FNB) for solid pancreatic lesions have been inconclusive with no clear superiority. The aim of this meta-analysis was to compare the diagnostic accuracy and safety between the two sampling techniques. Methods: We performed a systematic search of randomized controlled trials published between 2012 and 2019 . The primary outcome was overall diagnostic accuracy. Secondary outcomes included adverse event rates, cytopathologic and histopathologic accuracy, and the mean number of passes required to obtain adequate tissue between FNA and FNB needles. Fixed and random effect models with pooled estimates of target outcomes were developed.

Results: Eleven studies involving 1,365 participants were included for analysis. When compared to FNB, FNA had a significant reduction in diagnostic accuracy $(81 \%$ and $87 \%, p=0.005)$. In addition, FNA provided reduced cytopathologic accuracy $(82 \%$ and $89 \%, p=0.04)$ and an increased number of mean passes required compared to FNB (2.3 and 1.6, respectively, $p<0.0001)$. There was no difference in adverse event rate between FNA and FNB needles ( $1.8 \%$ and $2.3 \%$ respectively, $p=0.64)$.

Conclusions: FNB provides superior diagnostic accuracy without compromising safety when compared to FNA. FNB should be readily considered by endosonographers when evaluating solid pancreatic masses. Clin Endosc 2021;54:261-268

Key Words: Fine needle aspiration; Fine needle biopsy; Solid pancreatic lesions

\section{INTRODUCTION}

Pancreatic malignancy portends poor prognosis with a dismal 5-year survival of $8 \% .{ }^{1}$ Moreover, the disease burden of pancreatic cancer continues to rise, having the second highest

Received: April 17, 2020 Revised: May 8, 2020

Accepted: June 2, 2020

Correspondence: Benjamin D. Renelus

Division of Gastroenterology and Hepatobiliary Disease, New York-Presbyterian Brooklyn Methodist Hospital, 506 6th St. Brooklyn, NY 11215, USA

Tel: +1-863-521-4574, Fax: +1-718-780-3478, E-mail: brenelus@gmail.com

ORCID: https://orcid.org/0000-0001-5399-1483

(c) This is an Open Access article distributed under the terms of the Creative Commons Attribution Non-Commercial License (http://creativecommons.org/ licenses/by-nc/3.0) which permits unrestricted non-commercial use, distribution, and reproduction in any medium, provided the original work is properly cited. incidence among gastrointestinal tumors in the U.S. ${ }^{2}$ Prompt tissue diagnosis allows for early targeted treatment, potentially improving progression-free survival. ${ }^{3}$ The advent of endoscopic ultrasound-guided fine needle aspiration (EUS-FNA) and endoscopic ultrasound-guided fine needle biopsy (EUSFNB) has permitted minimally invasive tissue acquisition for tissue diagnosis. Unlike FNA needles, the reverse or opposing cutting bevel design of the FNB needle allows for the biopsy of core histopathologic tissue. ${ }^{4}$

EUS-FNA and EUS-FNB provide accurate diagnoses of solid pancreatic lesions with a reported sensitivity of $85 \%$ and $87 \%$, respectively. ${ }^{5,6}$ Previous studies comparing the two needles have yielded conflicting results with no clear superiority. ${ }^{7-10}$ There has been a recent increase in randomized con- 
trolled trials (RCTs) comparing the two techniques; however, there is a relative lack of updated meta-analyses examining clinical outcomes. This study aims to compare the clinical and technical outcomes between EUS-FNA and EUS-FNB when investigating solid pancreatic lesions.

\section{MATERIALS AND METHODS}

\section{Search strategy and eligibility criteria}

Two authors (BDR and DSJ) conducted an independent systematic electronic search of PubMed/Medline, Scopus, and Google Scholar databases for published articles comparing EUS-FNA and EUS-FNB between 01/01/2012 and $01 / 01 / 2019$. The search strategy was in accordance with the Preferred Reporting Items for Systematic Reviews and Meta-Analyses (PRISMA) guidance using a predefined protocol. ${ }^{11}$ With the assistance of an experienced health science librarian, the search terms used were EUS-FNA versus EUS-FNB AND pancreas OR pancreatic lesion. The aforementioned authors reviewed the study title and abstract for eligibility in the study. Disagreements were brought to the senior author (JMP) for final decision.

Studies were considered eligible if they met the following criteria: (1) RCT involving more than 30 live human participants; (2) published in the English language; and (3) provided data on the primary outcome, diagnostic accuracy. Studies were excluded if they were of a non-randomized design, involved animal or ex vivo studies, contained less than 30 participants, or lacked the outcome of interest.

\section{Data extraction and quality assessment}

Data abstraction was uniform for all studies and involved baseline characteristics and the outcomes of interests. To assess for clinical outcomes, records were collected of diagnostic accuracy and adverse event rate (AE). Technical success, histopathologic and cytopathologic accuracy, along with the number of passes required to obtain a diagnosis were obtained for technical outcomes. Study demographics and outcomes data can be viewed in Table 1. The Cochrane Collaboration's tool was used to assess the quality and risk of bias of selected studies. ${ }^{12}$ A Funnel plot was used to depict publication bias and Eggers regression test was used to quantitatively evaluate publication bias. ${ }^{13}$

\section{Outcomes analyzed}

Our primary outcome was diagnostic accuracy, defined as a proportion of overall accurate cytopathologic and histopathologic tissue diagnoses established within the attempted

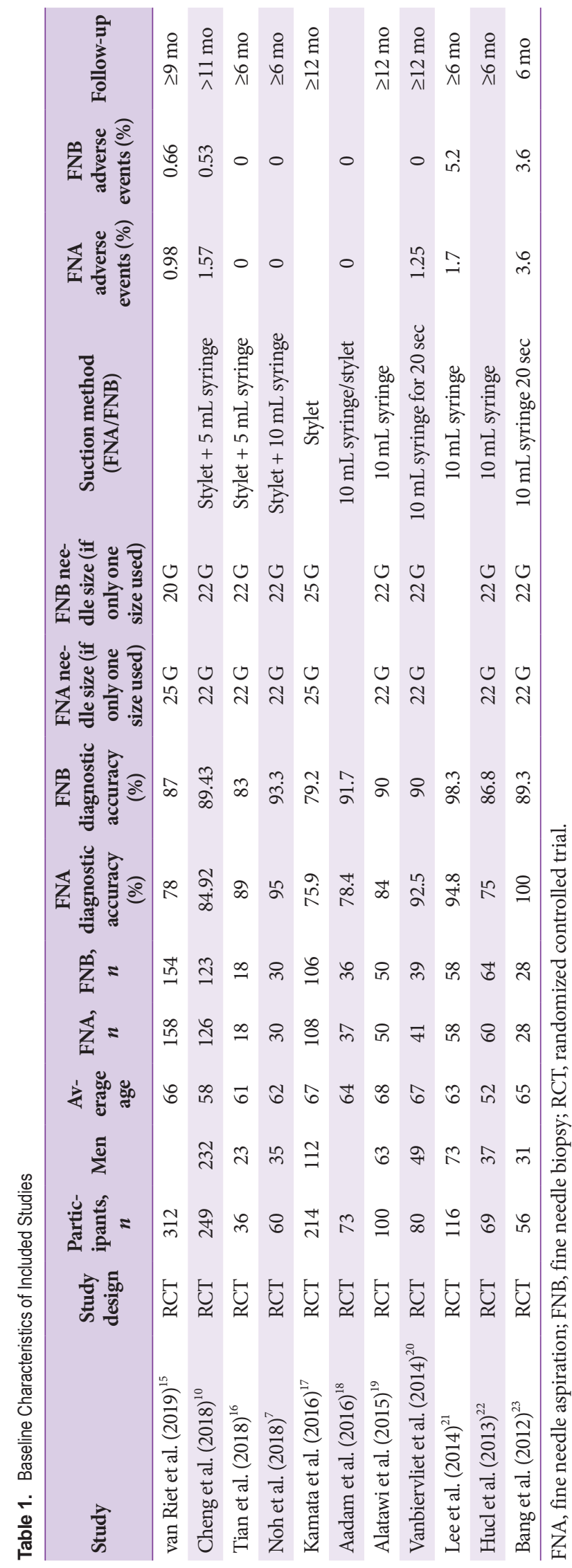


number of passes. The secondary outcomes included AE; cytopathologic accuracy, defined as greater than $50 \%$ of target cells present; histopathologic accuracy, defined as presence of histopathologic architecture and tissue core; the mean number of needle passes to achieve adequate tissue diagnosis; and technical success, defined as the ability to obtain a tissue sample.

\section{Statistical analysis}

Forest plots containing fixed and random effect models were generated for each outcome of interest. The Mantel-Haenszel method was used to compare proportions between device outcomes, with $p$-value $<0.05$ chosen for the level of significance. For the calculation of pooled overall means of numerical values from individual studies, we used the inverse variance method. We also computed the differences in pooled means between outcomes of interest and assessed the $p$-values of this difference. Heterogeneity between studies was tested using the $I^{2}$ statistics of Higgins. ${ }^{14}$ An $I^{2}>50 \%$ with a $p$-value $<0.05$ was considered statistically significant and prompted interpretation from the random effect model. We used the meta package version 4.9-1 for $\mathrm{R}$, and version 3.5.2 for all analyses ( $\mathrm{R}$ Foun- dation for Statistical Computing, Vienna, Austria). This study received approval from the institutional review board (IRB no. 618834-21). We received no funding for this study.

\section{RESULTS}

\section{Studies included}

We initially identified 257 records after duplicates were removed with our search strategy. After screening titles and abstracts, 236 articles were subsequently excluded. Six studies were also removed due to their retrospective or observational design, and 3 lacked the outcome of interest. One study was excluded due to the lack of a full manuscript. In total, 11 studies involving 1,365 patients were included for analysis (Fig. 1). ${ }^{7,10,15-23}$

The van Riet et al. ${ }^{15}$ used a novel $20 \mathrm{G}$ FNB needle while the Kamata et al. ${ }^{17}$ used a $25 \mathrm{G}$ core needle. The remaining studies used either 22 G FNB needles alone or multiple FNB needle sizes. Both the Kamata et al. and van Riet et al. used 25 G FNA needles, with the remaining studies using 22 G FNA or mul-



Records from PubMed/Medline/Scopus ( $n=252)$



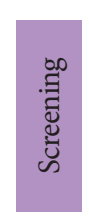
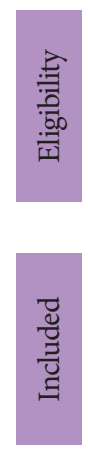

Number of records after duplicates removed


Fig. 1. Study selection flow diagram. 
tiple needle sizes. ${ }^{15,17}$ A total of 714 patients underwent FNA and 706 underwent FNB pancreatic tissue sampling.

\section{Primary and secondary endpoints}

The mean diagnostic accuracy for FNA and FNB was $81 \%$ and $87 \%$, respectively (pooled relative risk [RR], 0.94; $95 \%$ confidence interval [CI], 0.90-0.98; $p=0.005$ ) (Fig. 2). The mean cytopathologic accuracy for FNA and FNB was $82 \%$ and $89 \%$, respectively (pooled RR, 0.93; 95\% CI, 0.87-1.00; $p=0.04$ ) (Fig. 3). There was a numerical increase in the mean histopathologic accuracy of FNB compared to FNA but this finding did not reach significance $(81 \%$ and $74 \%$, respectively, pooled RR, 0.90; 95\% CI, 0.75-1.08; $p=0.39$ ) (Fig. 4). The mean technical success for FNA and FNB was $99 \%$ for both techniques (Fig. 5). FNB required almost one fewer needle passes to obtain adequate tissue compared to FNA (1.6 and 2.3 , respectively, mean difference, $0.71 ; 95 \%$ CI, 0.38-1.04; $p<0.0001$ ) (Fig. 6)



Fig. 2. Diagnostic accuracy. $\mathrm{Cl}$, confidence interval; FNA, fine needle aspiration; FNB, fine needle biopsy; RR, relative risk.



Fig. 3. Cytopathologic accuracy. $\mathrm{Cl}$, confidence interval; FNA, fine needle aspiration; FNB, fine needle biopsy; RR, relative risk. 




Fig. 4. Histopathologic accuracy. $\mathrm{Cl}$, confidence interval; FNA, fine needle aspiration; FNB, fine needle biopsy; RR, relative risk.

\begin{tabular}{|c|c|c|c|c|c|c|c|c|c|}
\hline Study & $n$ & $\begin{array}{l}\text { FNA } \\
\text { total }\end{array}$ & $n$ & $\begin{array}{l}\text { FNB } \\
\text { total }\end{array}$ & Risk ratio & $\mathbf{R R}$ & $95 \% \mathrm{CI}$ & $\begin{array}{l}\text { Weight } \\
\text { (fixed) }\end{array}$ & $\begin{array}{c}\text { Weight } \\
\text { (random) }\end{array}$ \\
\hline van Riet et al. (2019) ${ }^{15}$ & 158 & 158 & 152 & 154 & $\vdots$ & 1.01 & $0.99-1.03$ & $29.0 \%$ & $26.2 \%$ \\
\hline Cheng et al. $(2018)^{10}$ & 126 & 126 & 123 & 123 & + & 1.00 & $0.98-1.02$ & $23.5 \%$ & $34.6 \%$ \\
\hline Tian et al. $(2018)^{16}$ & 18 & 18 & 18 & 18 & $1 ;$ & 1.00 & $0.90-1.11$ & $3.5 \%$ & $0.7 \%$ \\
\hline Kamata et al. $(2016)^{17}$ & 108 & 108 & 106 & 106 & $\frac{1 i}{i}$ & 1.00 & $0.98-1.02$ & $20.2 \%$ & $25.6 \%$ \\
\hline Vanbiervliet et al. $(2014)^{20}$ & 41 & 41 & 39 & 39 & $\frac{1}{4}$ & 1.00 & $0.95-1.05$ & $7.6 \%$ & $3.6 \%$ \\
\hline Lee et al. $(2014)^{21}$ & 58 & 58 & 58 & 58 & i & 1.00 & $0.97-1.03$ & $11.0 \%$ & $7.5 \%$ \\
\hline Bang et al. $(2012)^{23}$ & 28 & 28 & 27 & 28 & $\frac{1}{3}$ & 1.04 & $0.97-1.11$ & $5.2 \%$ & $1.7 \%$ \\
\hline \multirow{2}{*}{\multicolumn{2}{|c|}{$\begin{array}{l}\text { Fixed effect model } \\
\text { Random effects model }\end{array}$}} & 537 & & 526 & $\dot{1}$ & 1.01 & $0.99-1.02$ & $100.0 \%$ & - \\
\hline & & & & & 8 & 1.00 & $0.99-1.01$ & - & $100.0 \%$ \\
\hline \multicolumn{5}{|c|}{ Heterogeneity: $I^{2}=0 \%, \tau^{2}=0, p=0.88$} & & & & & \\
\hline & & & & & 1 & & & & \\
\hline
\end{tabular}

Fig. 5. Technical success. Cl, confidence interval; FNA, fine needle aspiration; FNB, fine needle biopsy; RR, relative risk.

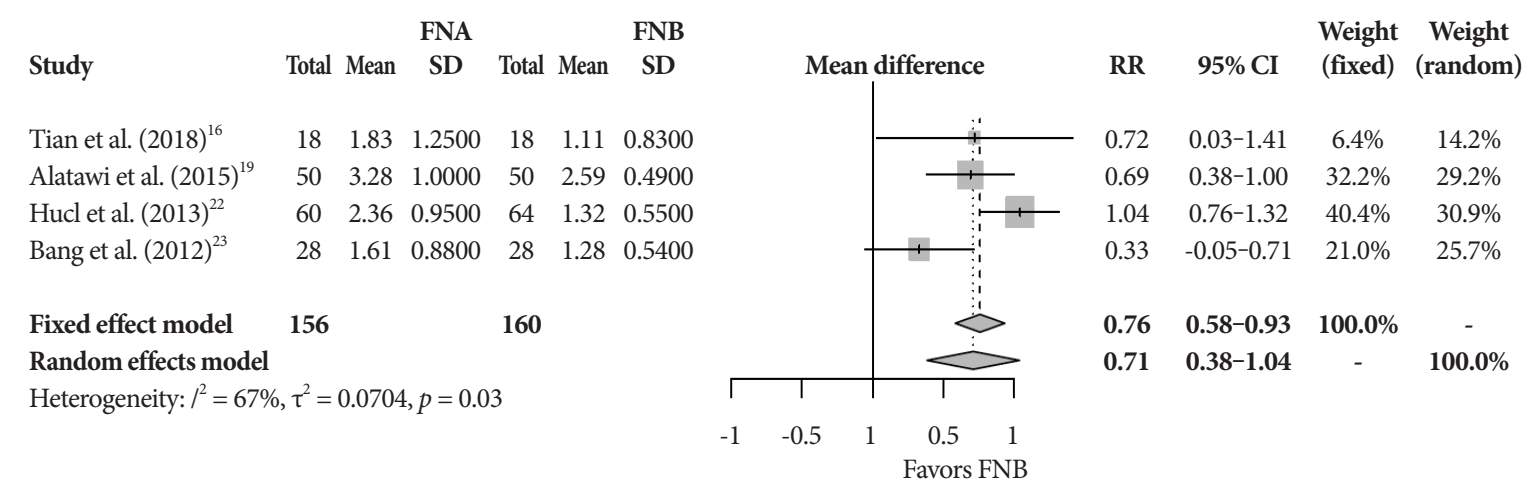

Fig. 6. Mean number of passes. $\mathrm{Cl}$, confidence interval; FNA, fine needle aspiration; FNB, fine needle biopsy; RR, relative risk; SD, standard deviation. 


\section{Adverse events and quality assessment}

There was no difference in the mean incident adverse events between FNB and FNA (2.3\% and 1.8\%, respectively, pooled
RR, 1.13; 95\% CI, 0.40-3.22; $p=0.64$ ) (Fig. 7). The Cochrane model showed a high-risk potential for bias in two studies, Aadam et al. in 2016 and Alatawi et al. in 2015 (Fig. 8). 18,19

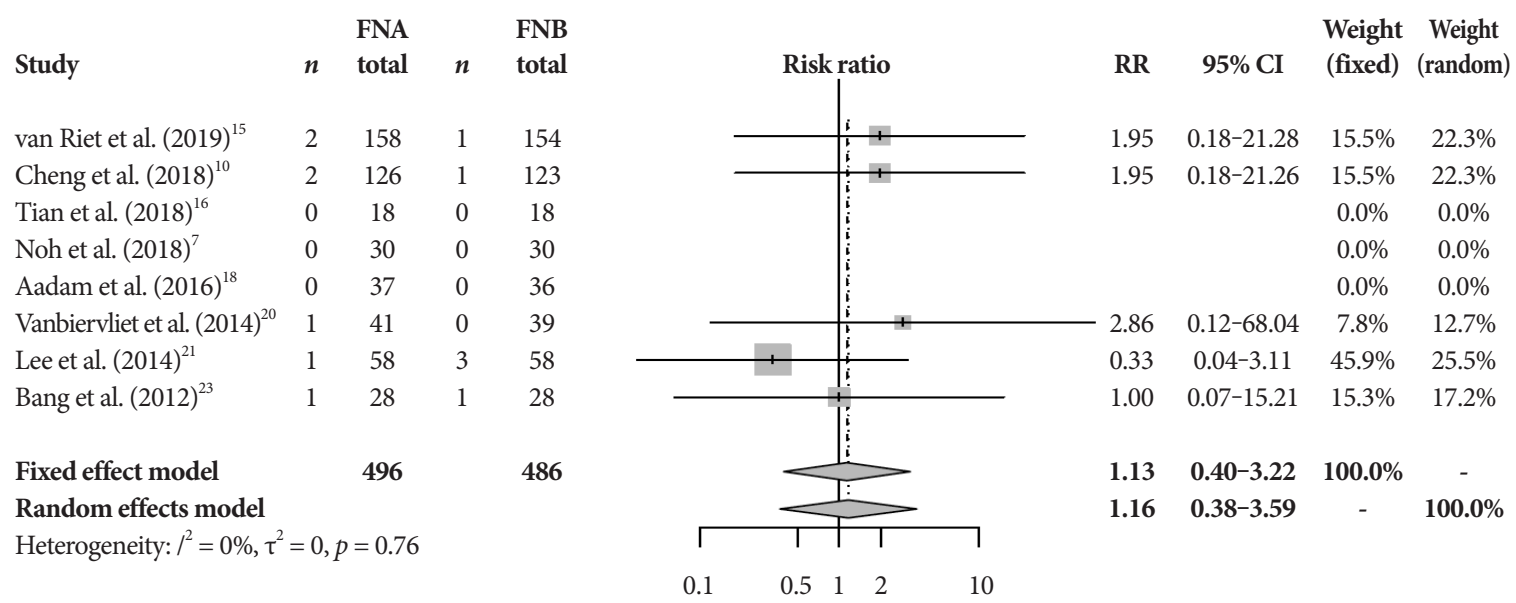

Fig. 7. Adverse event rate. $\mathrm{Cl}$, confidence interval; FNA, fine needle aspiration; FNB, fine needle biopsy; RR, relative risk.

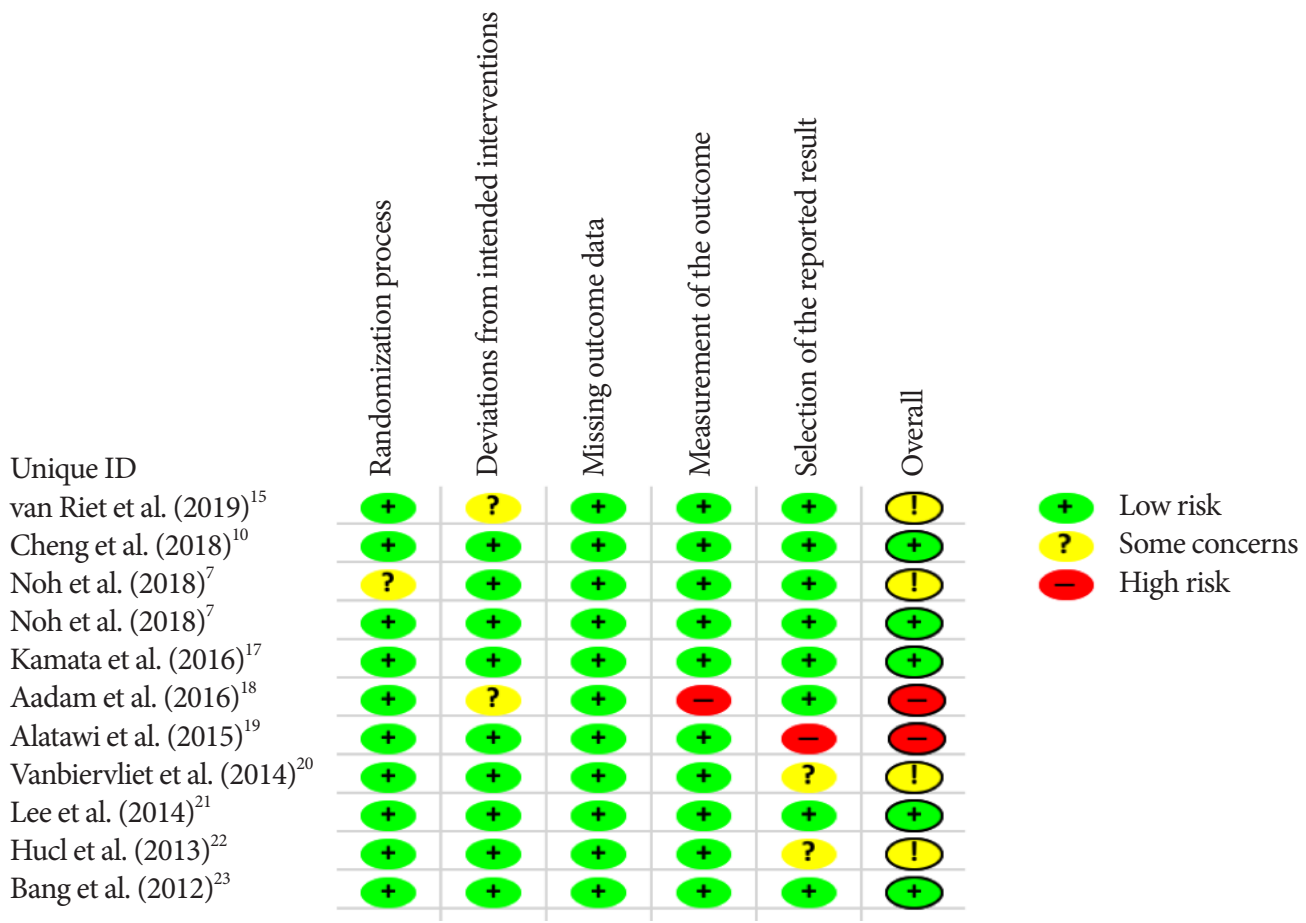

Fig. 8. Cochrane risk of bias. 


\section{DISCUSSION}

FNB provides superior diagnostic accuracy without compromising safety when compared to FNA. To our knowledge, this is the most robust meta-analysis on this topic, limited to RCT. Our findings are similar to those of Li et al. ${ }^{24}$ who also found that FNB provided superior diagnostic accuracy, specimen adequacy, and a reduced number of passes. However, cytopathologic differences were not reported in that study, although specimen adequacy was not defined and may be a surrogate for cytology. Furthermore, the authors included a prospective cohort study while we limited our data to RCTs. In their meta-analysis involving 8 RCT studies, Wang et al. ${ }^{25}$ found a reduced number of passes required to obtain tissue using FNB needles. The authors, however, did not find any difference in diagnostic accuracy.

FNB needles have been designed to obtain core tissue permitting preserved histological characteristics. Core tissue sampling enables the ability to diagnose pancreatic adenocarcinoma much like FNA, but also provides the ability to differentiate among other solid pancreatic lesions. ${ }^{26}$ Core tissue sampling can dramatically impact treatment by enabling molecular profiling which may be used for personalized medicine. Indeed, histopathologic specimens obtained from FNB yield an accurate pancreatic cancer diagnosis in over $90 \%$ of cases. ${ }^{5}$ Although we found that FNB provided greater cytopathologic accuracy when compared to FNA, there was only an insignificant increase in histopathologic accuracy with core/FNB needles. We acknowledge that in clinical practice tissue biopsy can be obtained with EUS-FNA and both cytology and histology are utilized to improve diagnostic accuracy. Furthermore, there are reports of suboptimal diagnostic yield with FNB in extra-pancreatic organs. Mohan et al. cite increased liver tissue specimens with FNA needles when compared to FNB needles in their $\mathrm{RCT}^{27}$ The authors also found no difference in diagnostic accuracy, technical success, or the number of passes required to obtain adequate diagnostic tissue. Never-the-less, the majority of publications comparing the two devices either show improvement in tissue acquisition with FNB or no difference between the two needles.

EUS-FNA has long been shown to provide accurate yet safer diagnostic capabilities when investigating pancreatic pathology compared to computed tomography guided biopsy. ${ }^{28}$ A concerns when using FNB is the potential increased risk of bleeding, as well as other complications. One way to address this concern is to provide fewer number of passes to obtain adequate tissue diagnosis. Our findings reveal a significant increase in the number of passes required to achieve adequate sampling with FNA compared to FNB. Increasing number of passes beyond 4 has not been shown to improve diagnostic yield in pancreatic cancer ${ }^{29}$ and may increase the risk of complications. ${ }^{30}$ Our findings showed a rare occurrence of AE, with no difference between FNA and FNB. Given the infrequence of $\mathrm{AE}$ and variations in reporting, we were unable to provide meaningful analysis regarding specific complications. Nevertheless, this study builds upon the safety of EUS-guided pancreatic tissue sampling irrespective of needle type.

There are currently three commercially available FNB needles. ${ }^{31}$ Facciorusso et al. group found that newer FNB needles, which have three symmetric cutting edges along the tip of the needle, were favorable compared to other FNB needles. ${ }^{32}$ Given the novelty, cost, and reliance upon operator experience, flexible FNB needles are often limited to robust referral centers.

There are notable limitations to our study. Firstly, we did not compare the various FNB needles due to the limited variations in comparative needle sizes in the studies analyzed. This is a growing area of interest as FNB needles are being utilized increasingly across medical centers. In addition, we were unable to analyze outcomes based upon the size and location of tumors due to inconsistent reporting. Moreover, potential bias was identified in our Cochrane model. Given this potential for confirmation bias, there is some concern that the results presented may be "too certain". This certainty cannot however be quantified numerically and must be acknowledged when interpreting our results.

Shortcomings notwithstanding, conducting a meta-analysis on high-quality studies with limited heterogeneity is one of the strengths of our study. To the best of our knowledge, this is the most comprehensive meta-analysis restricted to RCT broaching this topic. The findings of this study shed light on the strengths of FNB needle. EUS-FNB provides a significantly higher diagnostic yield with no difference in $\mathrm{AE}$ and thus, should be readily considered by endosonographers. As this area of gastroenterology continues to progress, further investigation is required to understand the meaningful differences between core needle biopsies.

Conflicts of Interest

The authors have no potential conflicts of interest.

Funding

None.

Author Contributions

Conceptualization: Benjamin D. Renelus, Daniel S. Jamorabo

Data curation: BDR, DSJ, Iman Boston

Formal analysis: William M. Briggs

Investigation: BDR, DSJ

Methodology: BDR, DSJ, WMB

Software: WMB

Supervision: John M. Poneros 
Visualization: WMB

Writing-original draft: BDR

Writing-review\&editing: BDR, DSJ, IB, WMB, JMP

ORCID

Benjamin D. Renelus:

Daniel S. Jamorabo:

Iman Boston:

William M. Briggs:

John M. Poneros:

https://orcid.org/0000-0001-5399-1483

https://orcid.org/0000-0001-7406-534X

https://orcid.org/0000-0003-1890-6495

https://orcid.org/0000-0001-8260-8244

https://orcid.org/0000-0003-1537-3101

\section{REFERENCES}

1. Siegel RL, Miller KD, Jemal A. Cancer statistics, 2018. CA Cancer J Clin 2018;68:7-30.

2. Siegel RL, Miller KD, Jemal A. Cancer statistics, 2019. CA Cancer J Clin 2019;69:7-34.

3. Kleeff J, Korc M, Apte M, et al. Pancreatic cancer. Nat Rev Dis Primers 2016;2:16022

4. Nayar MK, Paranandi B, Dawwas MF, et al. Comparison of the diagnostic performance of 2 core biopsy needles for EUS-guided tissue acquisition from solid pancreatic lesions. Gastrointest Endosc 2017;85:10171024.

5. Ishikawa T, Mohamed R, Heitman SJ, et al. Diagnostic yield of small histological cores obtained with a new EUS-guided fine needle biopsy system. Surg Endosc 2017;31:5143-5149.

6. Hewitt MJ, McPhail MJ, Possamai L, Dhar A, Vlavianos P, Monahan KJ. EUS-guided FNA for diagnosis of solid pancreatic neoplasms: a meta-analysis. Gastrointest Endosc 2012;75:319-331.

7. Noh DH, Choi K, Gu S, et al. Comparison of 22-gauge standard fine needle versus core biopsy needle for endoscopic ultrasound-guided sampling of suspected pancreatic cancer: a randomized crossover trial. Scand J Gastroenterol 2018;53:94-99.

8. Nagula S, Pourmand K, Aslanian H, et al. Comparison of endoscopic ultrasound-fine-needle aspiration and endoscopic ultrasound-fine-needle biopsy for solid lesions in a multicenter, randomized trial. Clin Gastroenterol Hepatol 2018;16:1307-1313.e1.

9. Hedenström P, Demir A, Khodakaram K, Nilsson O, Sadik R. EUS-guided reverse bevel fine-needle biopsy sampling and open tip fine-needle aspiration in solid pancreatic lesions - a prospective, comparative study. Scand J Gastroenterol 2018;53:231-237.

10. Cheng B, Zhang Y, Chen Q, et al. Analysis of fine-needle biopsy vs fine-needle aspiration in diagnosis of pancreatic and abdominal masses: a prospective, multicenter, randomized controlled trial. Clin Gastroenterol Hepatol 2018;16:1314-1321.

11. Liberati A, Altman DG, Tetzlaff J, et al. The PRISMA statement for reporting systematic reviews and meta-analyses of studies that evaluate health care interventions: explanation and elaboration. Ann Intern Med 2009;151:W65-W94.

12. Higgins JP, Altman DG, Gøtzsche PC, et al. The Cochrane collaboration's tool for assessing risk of bias in randomised trials. BMJ 2011;343:d5928.

13. Egger M, Davey Smith G, Schneider M, Minder C. Bias in meta-analysis detected by a simple, graphical test. BMJ 1997;315:629-634.

14. Higgins JP, Thompson SG. Quantifying heterogeneity in a meta-analysis. Stat Med 2002;21:1539-1558.

15. van Riet PA, Larghi A, Attili F, et al. A multicenter randomized trial comparing a 25-gauge EUS fine-needle aspiration device with a 20-gauge EUS fine-needle biopsy device. Gastrointest Endosc 2019;89:329-339.

16. Tian L, Tang AL, Zhang L, et al. Evaluation of $22 \mathrm{G}$ fine-needle aspiration
(FNA) versus fine-needle biopsy (FNB) for endoscopic ultrasound-guided sampling of pancreatic lesions: a prospective comparison study. Surg Endosc 2018;32:3533-3539.

17. Kamata K, Kitano M, Yasukawa S, et al. Histologic diagnosis of pancreatic masses using 25-gauge endoscopic ultrasound needles with and without a core trap: a multicenter randomized trial. Endoscopy 2016;48:632-638.

18. Aadam AA, Wani S, Amick A, et al. A randomized controlled cross-over trial and cost analysis comparing endoscopic ultrasound fine needle aspiration and fine needle biopsy. Endosc Int Open 2016;4:E497-E505.

19. Alatawi A, Beuvon F, Grabar S, et al. Comparison of $22 \mathrm{G}$ reverse-beveled versus standard needle for endoscopic ultrasound-guided sampling of solid pancreatic lesions. United European Gastroenterol J 2015;3:343352.

20. Vanbiervliet G, Napoléon B, Saint Paul MC, et al. Core needle versus standard needle for endoscopic ultrasound-guided biopsy of solid pancreatic masses: a randomized crossover study. Endoscopy 2014;46:10631070.

21. Lee YN, Moon JH, Kim HK, et al. Core biopsy needle versus standard aspiration needle for endoscopic ultrasound-guided sampling of solid pancreatic masses: a randomized parallel-group study. Endoscopy 2014;46:1056-1062.

22. Hucl T, Wee E, Anuradha S, et al. Feasibility and efficiency of a new $22 \mathrm{G}$ core needle: a prospective comparison study. Endoscopy 2013;45:792798.

23. Bang JY, Hebert-Magee S, Trevino J, Ramesh J, Varadarajulu S. Randomized trial comparing the 22-gauge aspiration and 22-gauge biopsy needles for EUS-guided sampling of solid pancreatic mass lesions. Gastrointest Endosc 2012;76:321-327.

24. Li H, Li W, Zhou QY, Fan B. Fine needle biopsy is superior to fine needle aspiration in endoscopic ultrasound guided sampling of pancreatic masses: a meta-analysis of randomized controlled trials. Medicine (Baltimore) 2018;97:e0207.

25. Wang J, Zhao S, Chen Y, Jia R, Zhang X. Endoscopic ultrasound guided fine needle aspiration versus endoscopic ultrasound guided fine needle biopsy in sampling pancreatic masses: a meta-analysis. Medicine (Baltimore) 2017;96:e7452.

26. Conti CB, Cereatti F, Grassia R. Endoscopic ultrasound-guided sampling of solid pancreatic masses: the fine needle aspiration or fine needle biopsy dilemma. Is the best needle yet to come? World J Gastrointest Endosc 2019;11:454-471.

27. Mohan BP, Shakhatreh M, Garg R, Ponnada S, Adler DG. Efficacy and safety of EUS-guided liver biopsy: a systematic review and meta-analysis. Gastrointest Endosc 2019;89:238-246.e3.

28. Okasha HH, Naga MI, Esmat S, et al. Endoscopic ultrasound-guided fine needle aspiration versus percutaneous ultrasound-guided fine needle aspiration in diagnosis of focal pancreatic masses. Endosc Ultrasound 2013;2:190-193.

29. Mohamadnejad M, Mullady D, Early DS, et al. Increasing number of passes beyond 4 does not increase sensitivity of detection of pancreatic malignancy by endoscopic ultrasound-guided fine-needle aspiration. Clin Gastroenterol Hepatol 2017;15:1071-1078.e2.

30. Erickson RA, Sayage-Rabie L, Beissner RS. Factors predicting the number of EUS-guided fine-needle passes for diagnosis of pancreatic malignancies. Gastrointest Endosc 2000;51:184-190.

31. Abdelfatah MM, Grimm IS, Gangarosa LM, Baron TH. Cohort study comparing the diagnostic yields of 2 different EUS fine-needle biopsy needles. Gastrointest Endosc 2018;87:495-500.

32. Facciorusso A, Del Prete V, Buccino VR, Purohit P, Setia P, Muscatiello N. Diagnostic yield of Franseen and fork-tip biopsy needles for endoscopic ultrasound-guided tissue acquisition: a meta-analysis. Endosc Int Open 2019;7:E1221-E1230. 